\title{
Neuromuscular Blocking Agents for ARDS: A Systematic Review and Meta-Analysis
}

\author{
Heather Torbic, Sudhir Krishnan, Mary Pat Harnegie, and Abhijit Duggal
}

\begin{abstract}
BACKGROUND: Studies evaluating neuromuscular blocking agents (NMBAs) in the management of ARDS have produced inconsistent results in terms of their effect on mortality. The purpose of this systematic review and meta-analysis was to evaluate differences in mortality comparing subjects with ARDS who received NMBA to those who received placebo or usual care. METHODS: We searched Ovid, MEDLINE, Embase, CINAHL, Cochrane, Scopus, and Web of Science for randomized controlled trials evaluating administration of NMBAs in subjects with ARDS. RESULTS: We included 6 studies $(N=1,558$ subjects $)$ from 1,814 abstracts identified by our search strategy. The use of early, continuous-infusion NMBAs reduces the risk of short-term (ie, 21-28-d) mortality (relative risk 0.71 [95\% CI $0.52-0.98], P=.030, I^{2}=60 \%$ ) in subjects with ARDS but does not reduce the risk of long-term (ie, 90-d) mortality (relative risk 0.81 [95\% CI 0.64-1.04], $P=.10, I^{2}=54 \%$ ). NMBAs decreased the risk of barotrauma (relative risk 0.55 [95\% CI $0.35-0.85], P=.008, I^{2}=0 \%$ ) and pneumothorax (relative risk 0.46 [95\% CI 0.28-0.77], $P=.003, I^{2}=0 \%$ ) compared to control. CONCLUSIONS: In subjects with ARDS, early use of NMBAs improves oxygenation, reduces the incidence of ventilator-induced lung injury, and decreases 21-28-d mortality, but it does not improve 90-d mortality. NMBAs should be considered for select patients with moderate-to-severe ARDS for short durations. Key words: acute respiratory distress syndrome; neuromuscular blocking agents; meta-analysis; paralysis; mortality; mechanical ventilation. [Respir Care 2021;66(1):120-128. (C) 2021 Daedalus Enterprises]
\end{abstract}

\section{Introduction}

ARDS is an acute inflammatory process that causes damage to the alveoli that impedes their ability to adequately oxygenate the body, thereby resulting in acute hypoxic respiratory failure. The initial treatment for

Dr Torbic is affiliated with the Department of Pharmacy, Cleveland Clinic, Cleveland, Ohio. Drs Krishnan and Duggal are affiliated with the Department of Critical Care, Respiratory Institute, Cleveland Clinic, Cleveland, Ohio. Ms Harnegie is affiliated with the Floyd D. Loop Alumni Library, Cleveland Clinic, Cleveland, Ohio.

Supplementary material related to this paper is available at http://www. rcjournal.com.

The authors have disclosed no conflicts of interest.

Correspondence: Heather Torbic PharmD BCPS BCCCP, Department of Pharmacy, Cleveland Clinic, 9500 Euclid Avenue, Hb-105, Cleveland, OH 44195. E-mail: torbich@ccf.org.

DOI: $10.4187 /$ respcare.07849
ARDS should include management of the underlying cause of ARDS and minimization of further injury. ${ }^{1-3}$ To date, nonpharmacologic treatment strategies such as lungprotective ventilation strategies minimizing inflation pressures and use of early prone position during ventilation to optimize recruitment have resulted in the greatest mortality benefit in patients with ARDS. Use of higher PEEP and conservative fluid management have been associated with better oxygenation and a higher number of ventilator-free days, respectively., ${ }^{1,2}$

Options for effective pharmacologic therapies in ARDS remain limited. ${ }^{4}$ Studies evaluating pharmacologic agents, either as early adjuncts or as rescue therapies, have demonstrated futility. ${ }^{4}$ Use of neuromuscular blocking agents (NMBAs) is the only studied pharmacologic treatment strategy that has demonstrated a mortality benefit in patients with early, severe ARDS. NMBAs are thought to provide benefit in ARDS by increasing recruitment and improving oxygen delivery and oxygen consumption matching. By paralyzing patients, ventilator asynchrony is minimized. This reduces ventilator-induced lung injury 


\section{Neuromuscular Blocking Agents in ARDS}

(VILI) and decreases circulating inflammatory mediators. ${ }^{5-7}$

Use of NMBAs in patients with ARDS has been consistently associated with improved oxygenation and decreased levels of inflammatory markers in clinical studies. ${ }^{8-13}$ However, an associated mortality benefit with these outcomes has been inconsistent, leading to variability in prescriber adoption of NMBA use in early, moderate-to-severe ARDS, with a recent study reported only $22.3 \%$ of subjects with early, moderate-to-severe ARDS received NMBAs. ${ }^{14,15}$ Given the results of the recently published Reevaluation of Systemic Early Neuromuscular Blockade (ROSE) trial, ${ }^{13}$ we sought to complete a systematic review and meta-analysis including these new data to evaluate subjects with early, moderate-to-severe ARDS receiving NMBAs as a primary intervention compared to placebo or usual care on 90-d mortality. We hypothesized that there would be no difference in 90-d mortality when using NMBAs in early, moderate-tosevere ARDS compared to usual care or placebo.

\section{Methods}

\section{Data Sources}

A systematic search of existing, relevant literature was performed by the authors, including an experienced medical information specialist, in the following databases: MEDLINE, Embase, Web of Science, Scopus, CINAHL, and Cochrane. The databases were searched from inception to May 25, 2019. Three elements were used in the search strategies: adult respiratory distress syndrome, neuromuscular blocking agents, and randomized clinical trials. These 3 elements were searched using controlled vocabulary, when available in the databases, and text searches to obtain results from PubMed and "text word only" databases. The complete search strategy can be found online (see the supplementary materials at http://www.rcjournal.com). The articles were imported in the reference software Endnote and then exported to the systematic review management software Covidence and checked for duplicates. Our systematic review and meta-analysis is registered in PROSPERO (CRD4202150538).

\section{Study Selection}

The titles/abstracts of identified studies were screened for full-text review by 2 independent study investigators (HT, AD). Studies, regardless of published language, were included for full-text review if they evaluated the use of NMBAs in subjects $\geq 18$ y old with ARDS. Full-text studies were then independently reviewed by 2 study investigators (HT, AD). Studies were included in the meta-analysis if they were randomized controlled studies comparing NMBAs to placebo or usual care in mechanically ventilated adult subjects with moderate-to-severe ARDS according to any ARDS definition and reported 28-d or 90-d mortality. No restrictions were placed on type or duration of continuous infusion or intermittent intravenous NMBA. We excluded case reports, case series, observational or retrospective studies, and studies evaluating the use of NMBAs in adult subjects with moderate-to-severe ARDS. Studies that did not compare NMBAs to placebo or usual care were also excluded.

\section{Data Extraction}

Relevant information from each study was selected and entered into a database in duplicate by 2 independent investigators (HT, AD): study design, sample size, study location, inclusion and exclusion criteria, and methodology. We also extracted data for all predefined end points of interest, including 90-d mortality (primary outcome), 28-d and ICU mortality, length of ICU and hospital stay, duration of mechanical ventilation, ventilator-free days, safety outcomes (eg, barotrauma, pneumothorax, ICU-acquired weakness), and ventilation parameters $\left(\mathrm{P}_{\mathrm{aO}_{2}} / \mathrm{F}_{\mathrm{IO}_{2}}\right.$ ratio, PEEP, plateau pressure). We chose 90-d mortality as the primary outcome because it was the primary end point in the 2 largest trials included in our meta-analysis. ${ }^{10,13}$

\section{Risk of Bias and Quality Assessment}

The Cochrane Risk of Bias tool ${ }^{16}$ was applied to each trial to evaluate the methodology for randomization, concealment, blinding, completeness of data, and selection outcome reporting. Each of these domains were assessed for low risk of bias, high risk of bias, or some concerns. Authors of included studies were not contacted to clarify domains of bias that we rated as having some concerns. Two independent authors (HT, AD) assessed the methodological quality of articles. Based upon study design and methodological quality, each individual study received an overall risk of bias according to the Cochrane Risk of Bias tool. A level of conclusion was determined after grouping studies with comparable methods, accounting for the study designs and the risk of bias. Domains in which studies were ranked as having a high risk of bias or some concerns were evaluated to assess the risk of bias to the overall results of the study. Studies that had $>4$ domains of bias with some concerns were labeled as having high overall risk of bias. Given the limitations in blinding when studying NMBAs, this domain was not heavily weighted when assessing overall risk of bias.

The 2 independent authors (HT, AD) also used the Grading of Recommendations, Assessment, Development, and Evaluation (GRADE) framework ${ }^{17}$ to evaluate the quality of evidence included in our pooled analysis across the domains of risk of bias, inconsistency, indirectness, 
imprecision, and publication bias, and overall quality of evidence was assigned to outcomes of interest after consensus between the 2 reviewing authors.

\section{Statistical Analysis}

RevMan 5.3 (Cochrane Review Manager Software; Nordic Cochrane Center, Copenhagen, Denmark) was used to pool data, and the DerSimonian Laird methods for random effects models were applied. ${ }^{18}$ Relative risk was calculated for dichotomous outcomes, and mean differences were calculated for continuous outcomes, both calculated with $95 \%$ CIs. The Mantel-Haenszel chi-square statistic was used to assess heterogeneity between studies, where $P<.01$ indicated significant heterogeneity and $I^{2}>50 \%$ indicated significant heterogeneity. We were unable to assess for publication bias using a funnel plot or other statistical methods due to the number of studies included in our meta-analysis. ${ }^{19}$ We performed a subgroup analysis to evaluate the impact of NMBAs on mortality including only studies with low overall risk of bias.

\section{Results}

\section{Study Selection}

Our search strategy identified 1,814 possible references for inclusion in our analysis. After the removal of duplicate references, 1,499 references were available for screening. Ultimately, we identified 6 studies that met our inclusion criteria (Fig. 1; see the supplementary materials at http:// www.rcjournal.com) ${ }^{8-13}$

\section{Study Description}

Characteristics of the included studies are listed in Table 1. Of the included studies, 4 were conducted by the same research team in ICUs in France, one study was conducted in China, and the final study was performed in hospitals in the United States. A total of 1,558 subjects with early, moderate-to-severe ARDS were included in the 6 studies. Each of these studies evaluated a continuous infusion of cisatracurium, a benzylisoquinoline NMBA, compared to placebo or usual care for $48 \mathrm{~h}$, with the exception of 1 study that studied a continuous infusion of vecuronium, an aminosteroid NMBA. ${ }^{11}$ Short-term mortality (21-28 d) was reported in 5 studies, ${ }^{8-11,13}$ ICU mortality was reported in 4 studies, ${ }^{8-11,13}$ and long-term mortality (90 d) was reported in 4 studies. ${ }^{8-10,13}$ The incidence of ICU-acquired weakness and VILI was reported in 4 studies. $^{8-10,13}$ ICU-acquired weakness was determined by clinical assessment (not confirmed with electromyography exam) in 2 of the studies ${ }^{8,9}$ and by using the validated Medical Research Council scale ${ }^{20}$ to assess

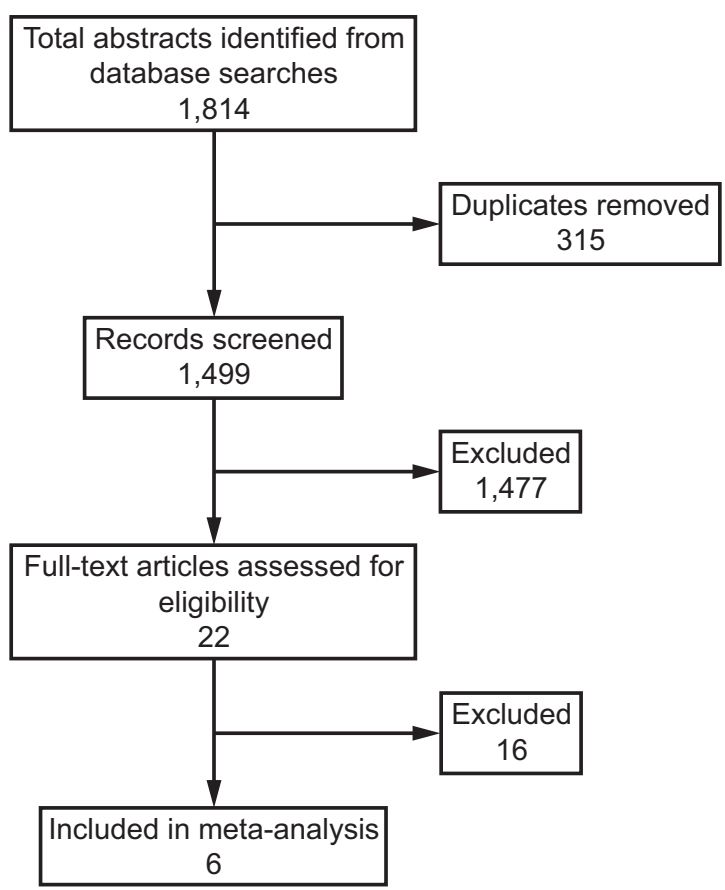

Fig. 1. Flow chart.

muscle strength in the other 2 studies. ${ }^{10,13}$ VILI was reported by clinicians in all studies; only 1 study ${ }^{10}$ set a standard definition for barotrauma by including any new pneumothorax, pneumomediastinum, subcutaneous emphysema, or pneumatocele $>2 \mathrm{~cm}$ in diameter.

\section{Risk of Bias and Study Quality}

Risk of bias of the included studies was determined using the Cochrane Risk of Bias tool ${ }^{16}$ and listed in Table 2. Of the included studies, 2 trials were deemed to have some concerns of bias due to a lack of blinding; given the effect of paralysis, blinding in the control arm would have been difficult and unlikely to affect final outcomes based on overall study methodology and data analysis. ${ }^{8}{ }^{89}$ Two studies were rated as having high risk of bias due to having $>4$ domains of bias with some concerns. ${ }^{11,12}$ Two studies were deemed to have low risk of bias due to overall low level of biases in their methodology and data analyses. ${ }^{10,13}$

Quality of the evidence for outcomes of interest was determined using the GRADE assessment tool (see the supplementary materials at http://www.rcjournal.com). ${ }^{17}$ The majority of evaluated outcomes were rated as having moderate-quality evidence to support findings, with the exception of barotrauma, pneumothorax, and ICU-acquired weakness, which were rated as having low-quality evidence. 


\section{Neuromuscular Blocking Agents in ARDS}

Table 1. Characteristics of Included Trials

\begin{tabular}{|c|c|c|c|c|}
\hline $\begin{array}{c}\text { Study, Country, Site, } \\
\text { Subjects }\end{array}$ & Details & Experimental Intervention & Control Intervention & Ventilator Strategies \\
\hline Gainnier et $\mathrm{al}^{8}$ & $\begin{array}{l}\text { SAPS II: } 37.7 \pm 0.7 \text { vs } 37.6 \pm \\
\quad 0.6, P=\mathrm{NS}\end{array}$ & $\begin{array}{l}\text { Cisatracurium } 50 \mathrm{mg} \\
\text { bolus then } 5 \mu \mathrm{g} / \mathrm{kg} / \mathrm{min} \\
\text { CIVI } \times 48 \mathrm{~h}\end{array}$ & $\begin{array}{l}\text { Sodium chloride } 0.9 \% \\
\quad 4 \mathrm{~mL} / \mathrm{h} \times 48 \mathrm{~h}\end{array}$ & ARMA protocol ${ }^{3}$ \\
\hline France & ARDS (AECC) & Goal train of four $<1$ & & No weaning protocol \\
\hline $\begin{array}{l}4 \text { medical or mixed } \\
\text { ICUs }\end{array}$ & $\mathrm{P}_{\mathrm{aO}_{2}} / \mathrm{F}_{\mathrm{IO}_{2}}<150$ & & & Volume assist-control \\
\hline$N=56$ adults & $\begin{array}{l}\text { Enrolled }<36 \mathrm{~h} \text { after ARDS } \\
\text { onset } \\
\text { Baseline } \mathrm{P}_{\mathrm{aO}_{2}} / \mathrm{F}_{\mathrm{IO}_{2}}: 130 \pm 34 \\
\quad \text { vs } 119 \pm 31, P=\mathrm{NS}\end{array}$ & & & $\mathrm{V}_{\mathrm{T}}=6-8 \mathrm{~mL} / \mathrm{kg}$ \\
\hline Forel et $\mathrm{al}^{9}$ & $\begin{array}{l}\text { SAPS II: } 49 \pm 19 \text { vs } 47 \pm 15 \text {, } \\
\quad P=\text { NS }\end{array}$ & $\begin{array}{l}\text { Cisatracurium } 0.2 \mathrm{mg} / \mathrm{kg} \\
\text { bolus then } 5 \mu \mathrm{g} / \mathrm{kg} / \mathrm{min} \\
\text { CIVI } \times 48 \mathrm{~h}\end{array}$ & $\begin{array}{l}\text { Sodium chloride } 0.9 \% \\
\quad 4 \mathrm{~mL} / \mathrm{h} \times 48 \mathrm{~h}\end{array}$ & ARMA protocol ${ }^{3}$ \\
\hline France & ARDS (AECC) & Goal train of four $<1$ & & No weaning protocol \\
\hline 3 ICUs & $\mathrm{P}_{\mathrm{aO}_{2}} / \mathrm{F}_{\mathrm{IO}_{2}} \leq 200$ & & & Volume assist-control \\
\hline$N=36$ adults & $\begin{array}{l}\text { Enrolled }<48 \mathrm{~h} \text { after ARDS } \\
\text { onset } \\
\text { Intubated }<48 \mathrm{~h}\end{array}$ & & & $\mathrm{~V}_{\mathrm{T}}=4-8 \mathrm{~mL} / \mathrm{kg}$ \\
\hline Papazian et $\mathrm{al}^{10}$ & $\begin{array}{l}\text { SAPS II: } 50 \pm 16 \text { vs } 47 \pm 14 \\
\quad P=.15\end{array}$ & $\begin{array}{l}\text { Cisatracurium } 15 \mathrm{mg} \\
\text { bolus then } 37.5 \mathrm{mg} / \mathrm{h} \\
\text { CIVI } \times 48 \mathrm{~h}\end{array}$ & $\begin{array}{l}\text { Placebo infusion } \times \\
\quad 48 \mathrm{~h}\end{array}$ & ARMA protocol ${ }^{3}$ \\
\hline France & ARDS (AECC) & No train of four & & Weaning protocol \\
\hline 20 ICUs & $\mathrm{P}_{\mathrm{aO}_{2}} / \mathrm{F}_{\mathrm{IO}_{2}}<150$ & & & Volume assist-control \\
\hline$N=340$ adults & $\begin{array}{l}\text { Enrolled }<48 \mathrm{~h} \text { after ARDS } \\
\quad \text { onset } \\
\text { Baseline } \mathrm{P}_{\mathrm{aO}_{2}} / \mathrm{F}_{\mathrm{IO}_{2}}: 106 \pm 36 \\
\quad \text { vs } 115 \pm 41, P=.03\end{array}$ & & & $\mathrm{~V}_{\mathrm{T}}=6-8 \mathrm{~mL} / \mathrm{kg}$ \\
\hline Lyu et $\mathrm{al}^{11}$ & $\begin{array}{c}\text { APACHE II: } 18.20 \pm 3.59 \text { vs } \\
19.37 \pm 4.14, P=\mathrm{NS}\end{array}$ & $\begin{array}{l}\text { Vecuronium } 0.1 \mathrm{~m} / \mathrm{kg} \\
\text { bolus then } 0.05 \mathrm{mg} / \mathrm{kg} / \\
\mathrm{h} \text { infusion } \times 24-48 \mathrm{~h}\end{array}$ & Usual care & No ventilation protocol \\
\hline China & ARDS (Berlin) & & & Volume assist-control \\
\hline $1 \mathrm{ICU}$ & $\mathrm{P}_{\mathrm{aO}_{2}} / \mathrm{F}_{\mathrm{IO}_{2}}<150$ & & & $\mathrm{~V}_{\mathrm{T}}=4-8 \mathrm{~mL} / \mathrm{kg}$ \\
\hline$N=96$ adults & $\begin{array}{l}\text { Enrolled }<48 \mathrm{~h} \text { after ARDS } \\
\text { onset } \\
\text { Baseline } \mathrm{P}_{\mathrm{aO}_{2}} / \mathrm{F}_{\mathrm{IO}_{2}}: 14.95 \pm \\
26.97 \text { vs } 144.33 \pm 24.09 \\
P=\mathrm{NS}\end{array}$ & & & \\
\hline Guervilly et al ${ }^{12}$ & $\begin{array}{l}\text { SAPS II: } 47(37-54) \text { vs } \\
\quad 48 \text { (42-62), } P=.40\end{array}$ & $\begin{array}{l}\text { Cisatracurium } 15 \mathrm{mg} \\
\text { bolus then } 37.5 \mathrm{mg} / \mathrm{h} \\
\mathrm{CIVI} \times 48 \mathrm{~h}\end{array}$ & Usual care & ARMA protocol ${ }^{3}$ \\
\hline France & ARDS (Berlin) & No train of four & & No weaning protocol \\
\hline 2 ICUs & $\mathrm{P}_{\mathrm{aO}_{2}} / \mathrm{F}_{\mathrm{IO}_{2}} \leq 200$ & & & Volume assist-control \\
\hline$N=24$ adults & $\begin{array}{l}\text { Enrolled }<48 \mathrm{~h} \text { after ARDS } \\
\text { onset } \\
\text { Baseline } \mathrm{P}_{\mathrm{aO}_{2}} / \mathrm{F}_{\mathrm{IO}_{2}}: 158(131- \\
\quad 185) \text { versus } 150(121-187), \\
\quad P=.40\end{array}$ & & & $\mathrm{~V}_{\mathrm{T}}=6 \mathrm{~mL} / \mathrm{kg}$ \\
\hline PETAL Network $^{13}$ & $\begin{array}{l}\text { APACHE III: } 103.9 \pm 3.1 \\
\quad \text { versus } 104.9 \pm 3.1, \mathrm{p}=\mathrm{NS}\end{array}$ & $\begin{array}{l}\text { Cisatracurium } 15 \mathrm{mg} \\
\text { bolus then } 37.5 \mathrm{mg} / \mathrm{h} \\
\mathrm{CIVI} \times 48 \mathrm{~h}\end{array}$ & Usual care & ARMA protocol $^{3}$ \\
\hline
\end{tabular}


Neuromuscular Blocking Agents In ARDS

Table 1. Continued

\begin{tabular}{|c|c|c|c|c|}
\hline $\begin{array}{l}\text { Study, Country, Site, } \\
\text { Subjects }\end{array}$ & Details & Experimental Intervention & Control Intervention & Ventilator Strategies \\
\hline \multirow{5}{*}{$\begin{array}{l}\text { United States } \\
48 \text { ICUs } \\
N=1,006 \text { adults }\end{array}$} & ARDS (Berlin) & No train of four & & High PEEP \\
\hline & $\mathrm{P}_{\mathrm{aO}_{2}} / \mathrm{F}_{\mathrm{IO}_{2}}<150$ & & & Weaning protocol \\
\hline & $\begin{array}{l}\text { Enrolled }<48 \mathrm{~h} \text { after ARDS } \\
\text { onset }\end{array}$ & & & $\begin{array}{l}\text { Light sedation target for } \\
\text { controls }\end{array}$ \\
\hline & $\begin{array}{l}\text { Baseline } \mathrm{P}_{\mathrm{aO}} / \mathrm{F}_{\mathrm{IO}_{2}}: 98.7 \pm \\
27.9 \text { versus } 99.5 \pm 27.9 \\
\mathrm{p}=\mathrm{NS}\end{array}$ & & & Volume assist-control \\
\hline & & & & $\mathrm{V}_{\mathrm{T}}=6 \mathrm{~mL} / \mathrm{kg}$ \\
\hline
\end{tabular}

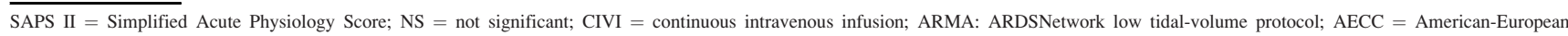
Consensus Conference; $\mathrm{V}_{\mathrm{T}}=$ tidal volume; APACHE $=$ Acute Physiology, Age, and Chronic Health Evaluation score

Table 2. Risk of Bias Assessment

\begin{tabular}{|c|c|c|c|c|c|c|c|c|}
\hline \multirow[b]{2}{*}{ Study } & \multicolumn{2}{|c|}{ Selection Bias } & \multirow{2}{*}{$\begin{array}{c}\text { Performance } \\
\text { Bias } \\
\text { Blinding } \\
\text { (Subjects and } \\
\text { Personnel) }\end{array}$} & \multirow{2}{*}{$\begin{array}{c}\text { Detection Bias } \\
\text { Blinding } \\
\text { (Outcome } \\
\text { Assessment) }\end{array}$} & \multirow{2}{*}{$\begin{array}{l}\text { Attrition } \\
\text { Bias } \\
\text { Incomplete } \\
\text { Outcome } \\
\text { Data }\end{array}$} & \multirow{2}{*}{$\begin{array}{c}\begin{array}{c}\text { Reporting } \\
\text { Bias }\end{array} \\
\begin{array}{c}\text { Selective } \\
\text { Reporting }\end{array}\end{array}$} & \multirow{2}{*}{$\begin{array}{c}\text { Other Bias } \\
\begin{array}{c}\text { Other Sources } \\
\text { of Bias }\end{array}\end{array}$} & \multirow[b]{2}{*}{ Overall Risk } \\
\hline & $\begin{array}{c}\text { Random } \\
\text { Sequence } \\
\text { Generation }\end{array}$ & $\begin{array}{l}\text { Allocation } \\
\text { Concealment }\end{array}$ & & & & & & \\
\hline Gainnier et $\mathrm{al}^{8}$ & Low & Low & High & High & Low & Low & Low & Some concerns \\
\hline Forel et $\mathrm{al}^{9}$ & Low & Low & High & High & Low & Low & Low & Some concerns \\
\hline Papazian et al ${ }^{10}$ & Low & Low & Low & Low & Low & Low & Low & Low \\
\hline Lyu et $\mathrm{al}^{11}$ & Some concerns & Some concerns & Some concerns & Some concerns & Low & Low & Some concerns & High \\
\hline Guervilly et $\mathrm{al}^{12}$ & Some concerns & Some concerns & Some concerns & Some concerns & Low & Low & Some concerns & High \\
\hline $\begin{array}{l}\text { PETAL } \\
\text { Network }^{13}\end{array}$ & Low & Low & Some concerns & Low & Low & Low & Low & Low \\
\hline
\end{tabular}

\section{Mortality}

Continuous-infusion NMBAs were associated with a decreased risk of short-term (21-28-d) mortality (relative risk 0.71 [95\% CI $0.52-0.98], P=.030, I^{2}=60 \%$ ) in subjects with early ARDS, but they did not reduce the risk of longterm hospital (90-d) mortality (relative risk $0.81,95 \%$ CI $0.64-1.04, P=.10, I^{2}=54 \%$ ). The use of NMBAs was also associated with a decreased risk of ICU mortality (relative risk $0.72,95 \%$ CI $0.57-0.91, P=.007, I^{2}=0 \%$ ) (Fig. 2).

\section{Safety}

In terms of VILI, NMBAs reduced the risk of barotrauma (relative risk $0.55,95 \%$ CI $0.35-0.85, P=.008, I^{2}=0 \%$ ) and pneumothorax (relative risk $0.46,95 \%$ CI $0.28-0.77$, $P=.003, I^{2}=0 \%$ ) compared to placebo or usual care. The use of NMBAs was not associated with an increased risk of ICU-acquired weakness (relative risk 1.15, 95\% CI 0.95$\left.1.39, P=.16, I^{2}=0 \%\right)$ (Fig. 3).

\section{Oxygenation and Ventilator Requirements}

Improvement in oxygenation was assessed via change in the $\mathrm{P}_{\mathrm{aO}_{2}} / \mathrm{F}_{\mathrm{IO}_{2}}$ ratio, which was reported in 4 studies at 24-, 48-, and 72-h after randomization time points. Subjects who received NMBAs did not have improved oxygenation at 24-h after randomization $(P=.19)$, but they did have improved oxygenation at 48 -h $(P=.01)$ and 72 -h $(P=$ $.030)$ after randomization compared to controls. The use of NMBAs was not associated with a decreased need for mechanical ventilation because duration of mechanical ventilation was unchanged between control subjects and subjects who received NMBAs, for all subjects $(P=.43)$ and survivors $(P=.93)$. Ventilator-free days were reported in 4 studies and also did not differ between subjects who received NMBAs and control subjects (relative risk 0.86, $95 \%$ CI $-0.61-2.33, P=.25, I^{2}=34 \%$ ). NMBA use was also not associated with improvements in plateau pressure or the use of higher PEEP (see the supplementary materials at http://www.rcjournal.com). 
Neuromuscular Blocking Agents In ARDS

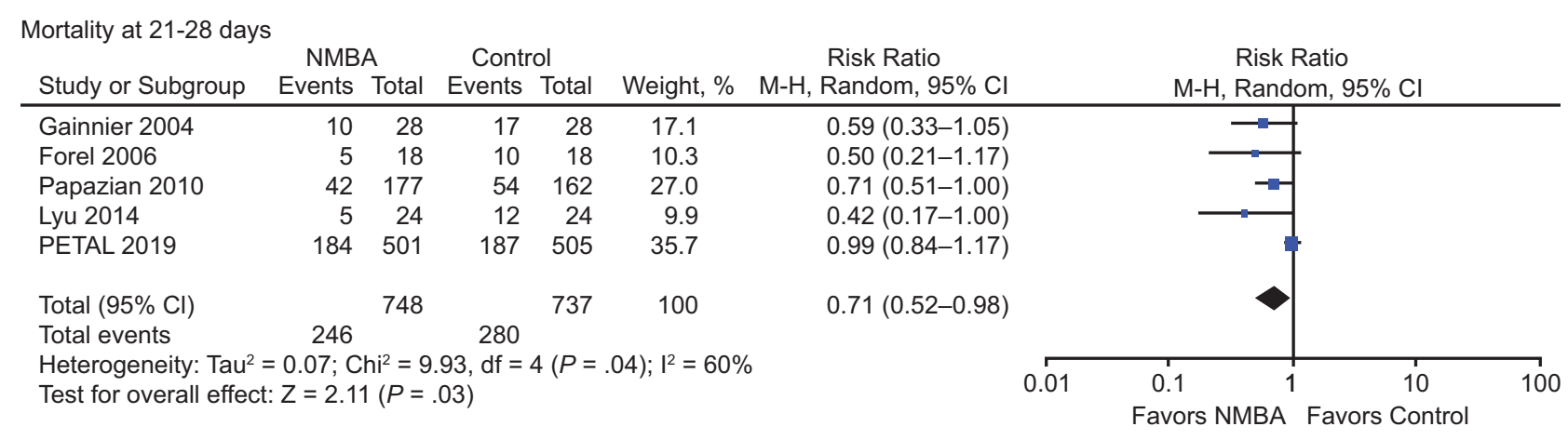

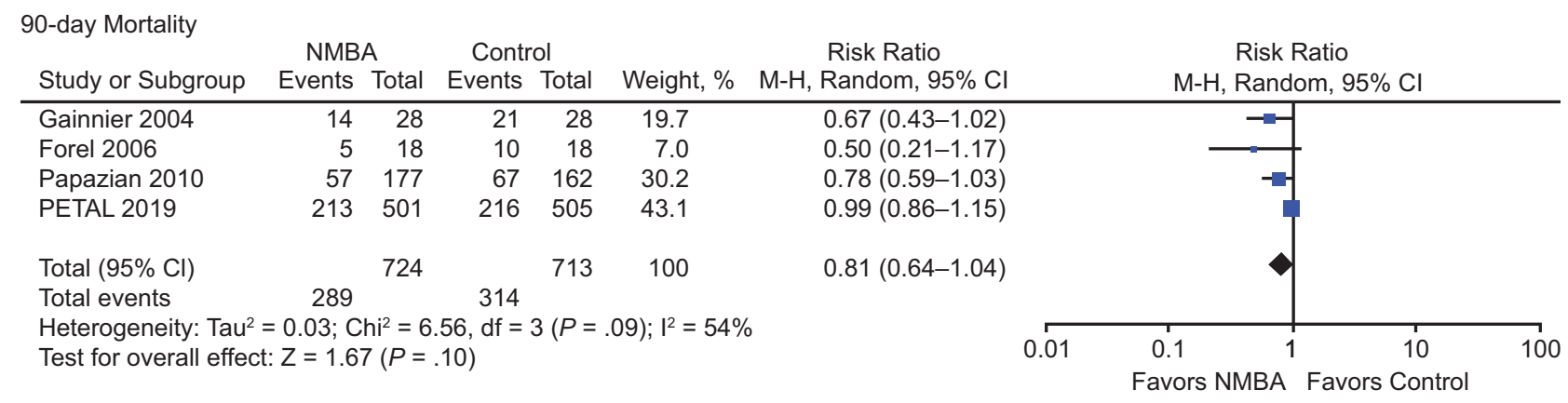

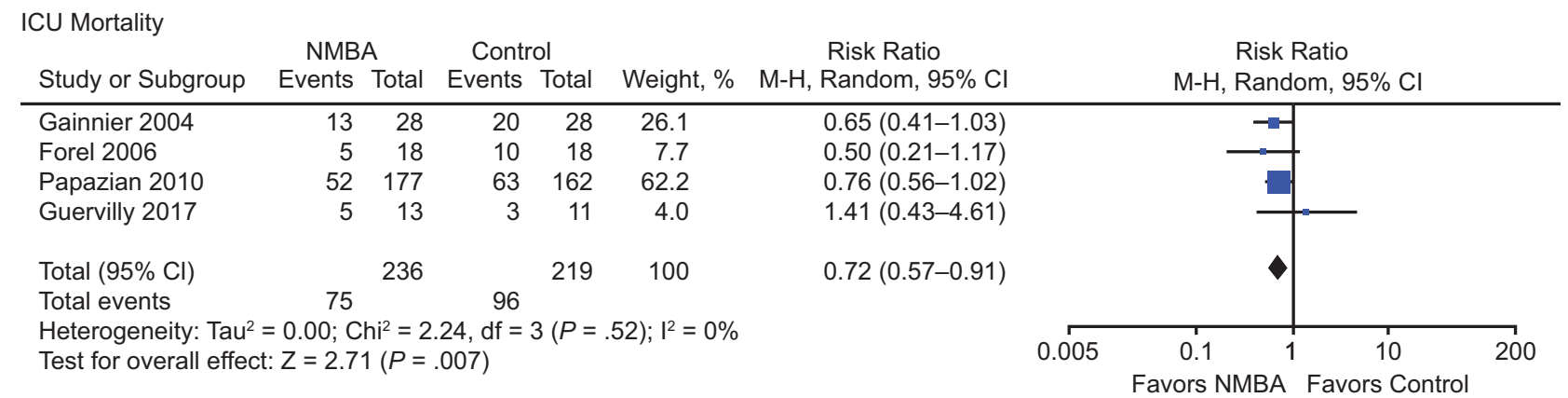

Fig. 2. Effect of neuromuscular blocking agents (NMBAs) on 21-28-d mortality, 90-d mortality, and ICU mortality.

\section{Subgroup Analysis}

When only evaluating 21-28-d mortality in studies with a low risk of bias, NMBAs no longer provided a mortality benefit (relative risk $0.87,95 \%$ CI $0.63-1.20, P=$ $.39, I^{2}=66 \%$ ). The risk of 90 -d mortality also remained nonsignificant in this subgroup (relative risk $0.91,95 \% \mathrm{CI}$ $0.72-1.14, P=.41, I^{2}=56 \%$ ) (see the supplementary materials at http://www.rcjournal.com).

\section{Discussion}

In this meta-analysis, we found that $48 \mathrm{~h}$ of NMBA therapy in subjects with early, moderate-to-severe ARDS is associated with a decrease in 21-28-d mortality but has no effect on 90-d mortality. NMBAs also reduce the incidence of VILI and do not increase the risk of ICU-acquired weakness. Short-term use of NMBAs in subjects with early ARDS improves oxygenation but does not decrease duration of mechanical ventilation. The results of our metaanalysis are similar to previous meta-analyses that included 3 and 5 studies, respectively, ${ }^{21,22}$ but in light of the ROSE ${ }^{13}$ results, our findings differ with regard to 90-d mortality.

The results of our meta-analysis include the results of the ROSE trial, the largest trial to date, which reported no mortality benefit associated with NMBA use at $90 \mathrm{~d}$ regardless of ARDS severity or duration..$^{13}$ These results differ from the ACURASYS trial, which reported a 90-d mortality benefit associated with NMBA use. ${ }^{10}$ ROSE and ACURASYS account for $86 \%$ of the subjects assessed in this meta-analysis, and both are methodologically sound and associated with a low risk of bias and moderate quality of evidence. ${ }^{10,13}$ Our meta-analysis noted that NMBAs improved 
Neuromuscular Blocking Agents In ARDS

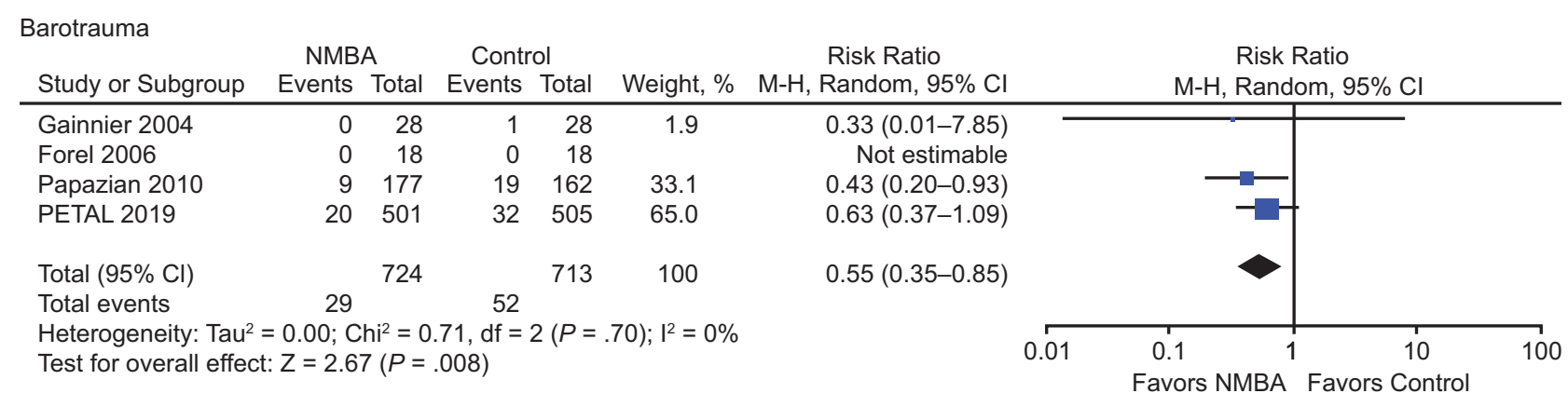

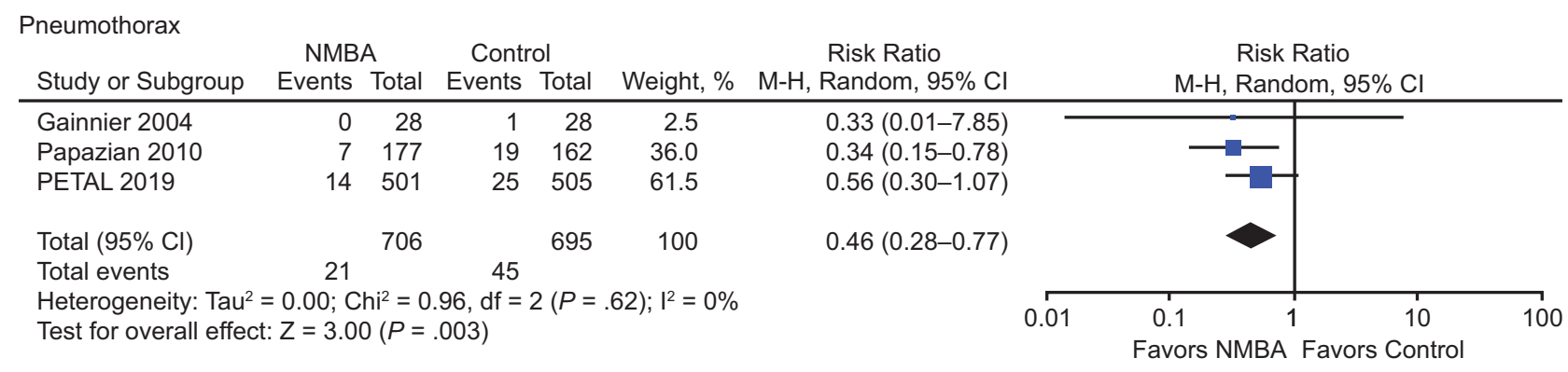

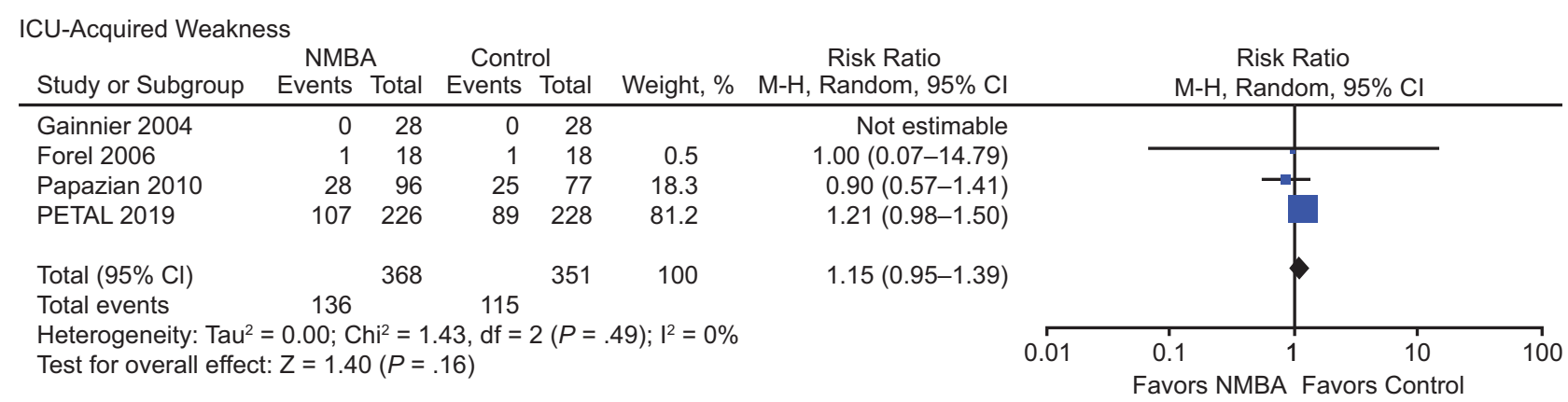

Fig. 3. Effect of neuromuscular blocking agents (NMBAs) on safety end points including barotrauma, pneumothorax, and ICU-acquired weakness.

21-28-d mortality, but there was moderate heterogeneity $\left(I^{2}=60 \%\right)$ among trials included in this analysis. Most likely, differences in methodology, particularly mechanical ventilation strategies such as incorporation of high PEEP, led to outcome variations among trials. ${ }^{8-11,13}$

Despite the strengths of the ACURASYS and ROSE trials, there are a few key differences between these trials that may have resulted in conflicting outcomes. First, in ACURASYS, both the control arm and the NMBA arm received deep sedation. ${ }^{10}$ In ROSE, subjects who were randomized to NMBAs received deep sedation, but for subjects in the control arm, light sedation was targeted and daily sedation interruptions were encouraged. ${ }^{13}$ Recent studies have shown that deeper levels of sedation in the early phases of ARDS can increase the likelihood of reverse triggering, a form of ventilator asynchrony that can result in breath-stacking, VILI, and increased mortality. ${ }^{23-25}$ Although both trials protocolized ventilator management and utilized lung-protective ventilation, the ROSE trial also used a high-PEEP strategy, ${ }^{10,13}$ which has been associated with improved recruitment and decreased lung stress and atelectrauma. ${ }^{2}$ There was no difference in PEEP when comparing control and NMBA subjects on days $0-7$ of both the ACURASYS and ROSE trials, ${ }^{10,13}$ suggesting the impact of NMBAs may be minimized when added to a high-PEEP strategy like that utilized in the ROSE trial. Another key difference was the time to enrollment between the ACURASYS and ROSE trials. The mean time to inclusion for subjects in ACURASYS was 21-22 h, whereas the median time to randomization for subjects enrolled in ROSE was 6.8-8.2 h. ${ }^{10,13}$ As previously reported, ${ }^{26}$ a subset of subjects with moderate-to-severe ARDS improve within 24 $\mathrm{h}$ of intubation, and just by virtue of the timing of enrollment, these subjects would be excluded from ACURASYS and would be randomized in ROSE. Despite the methodological differences between these 2 studies, the results of 


\section{Neuromuscular Blocking Agents in ARDS}

this meta-analysis highlight the importance of nonpharmacologic treatment. These approaches include utilizing PEEP optimization, lung-protective ventilation, and the addition of NMBAs in patients who require additional control of unrestrained and potentially dangerous patterns of spontaneous breathing, thereby reducing discomfort and demands for oxygen delivery and ventilation.

In our meta-analysis, use of NMBAs was associated with an improvement in the $\mathrm{P}_{\mathrm{aO}_{2}} / \mathrm{F}_{\mathrm{IO}_{2}}$ ratio at $48 \mathrm{~h}$ and $72 \mathrm{~h}$ after NMBA initiation. Plateau pressures did not improve with the use of NMBAs, and there was no significant difference in the utilization of PEEP with NMBA use. Additionally, improvements in oxygenation did not translate to decreased duration of mechanical ventilation or an increase in ventilator-free days. Subjects who received NMBAs had significantly less VILI, including barotrauma and pneumothorax. Currently, lung-protective ventilator strategies are recommended as the first-line of management to reduce the incidence of VILI in patients with ARDS by minimizing inflation pressures and providing recruitment. ${ }^{2,3,27}$ In the included studies in this meta-analysis, both the NMBA and control arms followed the same mechanical ventilation protocols, and subjects had similar tidal volumes and PEEP at baseline. Given that factors thought to be related to reduced incidence of VILI in patients with ARDS were similar at baseline in both the subjects who received NMBAs and those who received usual care, the anti-inflammatory properties of NMBAs may be the reason for decreased VILI. ${ }^{9,27}$

The use of NMBAs is not without risk. NMBA use has been associated with ICU-acquired weakness. A meta-analysis evaluating the use of NMBAs and associated neuromuscular dysfunction in critically ill subjects found an association (odds ratio $1.25,95 \%$ CI 1.06-1.48, $I^{2}=16 \%$ ), but no association was noted when evaluating only studies with a low risk of bias (odds ratio 1.21, 95\% CI 0.672.19). ${ }^{28}$ Similar to a prior meta-analysis, ${ }^{21}$ we found no increased incidence of ICU-acquired weakness in subjects with ARDS who received a short course of NMBA therapy. A recent study evaluating NMBA use in mechanically ventilated subjects with sepsis reported that NMBAs provided the greatest mortality benefit if they were used for $<2 \mathrm{~d}^{29}$ These findings, in addition to studies evaluating NMBAs in subjects with ARDS, suggests that short NMBA courses are superior for both efficacy and safety outcomes.

Our systematic review and meta-analysis has a few limitations. First, we did not contact authors of studies for additional data, and this meta-analysis was not completed using individual subject data. Additionally, we did not contact authors of studies that evaluated NMBAs for ARDS but did not report 21-28-d and 90-d mortality. Unfortunately, none of the reported outcomes in this meta-analysis were evaluated by all 6 included studies. This may have resulted in a loss of analyzable data, although we find it unlikely that this affected the overall findings of our analysis given the small amount of lost data. Despite these limitations, our meta-analysis has several strengths compared to prior meta-analyses. First, we completed a thorough literature search to identify applicable trials, and this is the largest meta-analysis to date in terms of including the greatest number of subjects with ARDS. We also performed multiple subgroup analyses to minimize any influence on the results from studies with a risk of bias or low-quality evidence.

It is clear that lung-protective ventilation, optimal titration of PEEP, and minimization of sedation should be actively targeted and instituted for all patients with ARDS. ${ }^{2}$ Currently, the use of NMBAs in patients with ARDS is given a weak recommendation in the guidelines published by the Society of Critical Care Medicine. ${ }^{30}$ Use of NMBAs helps achieve physiologic benefit in hypoxemic subjects and has a short-term mortality benefit, but this does not translate into long-term benefits. Due to the risks of deep sedation and prolonged neuromuscular blockade, we cannot use NMBAs as a front-line adjunct therapy in all patients, and patients should be individually evaluated by clinicians to assess whether NMBAs are physiologically indicated. Nonpharmacologic strategies utilizing lung-protective ventilation and PEEP optimization should continue to be the backbone of ARDS management. ${ }^{2}$ If patients with early, moderate-to-severe ARDS do not experience improvements in oxygenation or remain dysynchronous with the ventilator after $12 \mathrm{~h}$ of optimal nonpharmacologic treatment strategies, NMBAs should be considered. ${ }^{31}$

\section{Conclusions}

In subjects with moderate-to-severe ARDS, early use of NMBAs improves oxygenation, reduces the incidence of VILI, and decreases 21-28-d mortality, but it does not improve 90-d mortality. NMBAs should be considered for select patients with moderate-to-severe ARDS for short durations.

\section{REFERENCES}

1. Thompson BT, Chambers RC, Liu KD. Acute respiratory distress syndrome. N Engl J Med 2017;377(6):562-572.

2. Fan E, Del Sorbo L, Goligher EC, Hodgson CL, Munshi L, Walkey AJ, et al. An official American Thoracic Society/European Society of Intensive Care Medicine/Society of Critical Care Medicine clinical practice guideline: mechanical ventilation in adult patients with acute respiratory distress syndrome. Am J Respir Crit Care Med 2017;195 (9):1253-1263.

3. Brower RG, Matthay MA, Morris A, Schoenfeld D, Thompson BT, Wheeler A. Ventilation with lower tidal volumes as compared with traditional tidal volumes for acute lung injury and the acute respiratory distress syndrome. N Engl J Med 2000;342(18):1301-1308.

4. Duggal A, Ganapathy A, Ratnapalan M, Adhikari NK. Pharmacological treatments for acute respiratory distress syndrome: systematic review. Minerva Anestesiol 2015;81(5):567-588. 


\section{Neuromuscular Blocking Agents in ARDS}

5. Slutsky AS. Neuromuscular blocking agents in ARDS. N Engl J Med 2010;363(12):1176-1180.

6. Gattinoni L, Marini JJ. Prone positioning and neuromuscular blocking agents are part of standard care in severe ARDS patients: we are not sure. Intensive Care Med 2015;41(12):2201-2203.

7. Hraiech S, Forel JM, Papazian L. The role of neuromuscular blockers in ARDS: benefits and risks. Curr Opin Crit Care 2012;18(5):495-502.

8. Gainnier M, Roch A, Forel JM, Thirion X, Arnal JM, Donati S, et al. Effect of neuromuscular blocking agents on gas exchange in patients presenting with acute respiratory distress syndrome. Crit Care Med 2004;32(1):113-119.

9. Forel JM, Roch A, Marin V, Michelet P, Demory D, Blache JL, et al. Neuromuscular blocking agents decrease inflammatory response in patients presenting with acute respiratory distress syndrome. Crit Care Med 2006;34(11):2749-2757.

10. Papazian L, Forel JM, Gacouin A, Penot-Ragon C, Perrin G, Loundou A, et al. Neuromuscular blockers in early acute respiratory distress syndrome. N Engl J Med 2010;363(12):1107-1116.

11. Lyu G, Wang X, Jiang W, Cai T, Zhang Y. [Clinical study of early use of neuromuscular blocking agents in patients with severe sepsis and acute respiratory distress syndrome]. Zhonghua Wei Zhong Bing Ji Jiu Yi Xue 2014;26(5):325-329.

12. Guervilly C, Bisbal M, Forel JM, Mechati M, Lehingue S, Bourenne J, et al. Effects of neuromuscular blockers on transpulmonary pressures in moderate to severe acute respiratory distress syndrome. Intensive Care Med 2017;43(3):408-418.

13. National Heart, Lung, and Blood Institute PETAL Clinical Trials Network, Moss M, Huang DT, Brower RG, Ferguson ND, Ginde AA, et al. Early neuromuscular blockade in the acute respiratory distress syndrome. N Engl J Med 2019;380(21):1997-2008.

14. Bellani G, Laffey JG, Pham T, Fan E, Brochard L, Esteban A, et al. Epidemiology, patterns of care, and mortality for patients with acute respiratory distress syndrome in intensive care units in 50 countries. JAMA 2016;315(8):788-800.

15. Duggal A, Rezoagli E, Pham T, McNicholas BA, Fan E, Bellani G, et al. Patterns of use of adjunctive therapies in patients with early moderate to severe ARDS: insights from the LUNG SAFE study. Chest 2020;157(6): 1497-1505.

16. Jørgensen L, Paludan-Müller AS, Laursen DRT, Savović J, Boutron I, Sterne JAC, et al. Evaluation of the Cochrane tool for assessing risk of bias in randomized clinical trials: overview of published comments and analysis of user practice in Cochrane and non-Cochrane reviews. Syst Rev 2016;5:80.

17. Balshem H, Helfand M, Schünemann HJ, Oxman AD, Kunz R, Brozek J, et al. GRADE guidelines: 3 . Rating the quality of evidence. J Clin Epidemiol 2011;64(4):401-406.
18. DerSimonian R, Laird N. Meta-analysis in clinical trials. Control Clin Trials 1986;7(3):177-188.

19. Egger M, Davey Smith G, Schneider M, Minder C. Bias in meta-analysis detected by a simple, graphical test. BMJ 1997;315(7109):629634.

20. De Jonghe B, Sharshar T, Lefaucheur JP, Authier FJ, Durand-Zaleski I, Boussarsar M, et al. Paresis acquired in the intensive care unit: a prospective multicenter study. JAMA 2002;288(22):2859-2867.

21. Alhazzani W, Alshahrani M, Jaeschke R, Forel JM, Papazian L, Sevransky J, et al. Neuromuscular blocking agents in acute respiratory distress syndrome: a systematic review and meta-analysis of randomized controlled trials. Crit Care 2013;17(2):R43.

22. Tao W, Yang LQ, Gao J, Shao J. Neuromuscular blocking agents for adult patients with acute respiratory distress syndrome: a meta-analysis of randomized controlled trials. J Trauma Acute Care Surg 2018;85 (6): 1102-1109.

23. Blanch L, Villagra A, Sales B, Montanya J, Lucangelo U, Luján M, et al. Asynchronies during mechanical ventilation are associated with mortality. Intensive Care Med 2015;41(4):633-641.

24. Akoumianaki E, Lyazidi A, Rey N, Matamis D, Perez-Martinez N, Giraud R, et al. Mechanical ventilation-induced reverse-triggered breaths: a frequently unrecognized form of neuromechanical coupling. Chest 2013;143(4):927-938

25. Slutsky AS, Villar J. Early paralytic agents for ARDS? Yes, no, and sometimes. N Engl J Med 2019;380(21):2061-2063.

26. Schenck EJ, Oromendia C, Torres LK, Berlin DA, Choi AMK, Siempos II. Rapidly improving ARDS in therapeutic randomized controlled trials. Chest 2019;155(3):474-482.

27. Slutsky AS, Ranieri VM. Ventilator-induced lung injury. N Engl J Med 2014;370(10):980.

28. Price DR, Mikkelsen ME, Umscheid CA, Armstrong EJ. Neuromuscular blocking agents and neuromuscular dysfunction acquired in critical illness: a systematic review and meta-analysis. Crit Care Med 2016;44(11):2070-2078

29. Steingrub JS, Lagu T, Rothberg MB, Nathanson BH, Raghunathan K, Lindenauer PK. Treatment with neuromuscular blocking agents and the risk of in-hospital mortality among mechanically ventilated patients with severe sepsis. Critical Care Med 2014;42(1):90-96.

30. Murray MJ, DeBlock H, Erstad B, Gray A, Jacobi J, Jordan C, et al. Clinical practice guidelines for sustained neuromuscular blockade in the adult critically ill patient. Crit Care Med 2016;44(11):2079-2103.

31. Guérin C, Reignier J, Richard JC, Beuret P, Gacouin A, Boulain T, et al. Prone positioning in severe acute respiratory distress syndrome. N Engl J Med 2013;368(23):2159-2168. 